Abstract ID: 24

Clinical Medicine

Oral

\title{
The Diagnostic and Predictive Value of Plasma Cystatin C in Acute Kidney Injury Secondary to Sepsis in The Intensive Care Unit
}

Iqbalmunauwir bin Ab Rashid ${ }^{1}$, Azrina Md Ralib ${ }^{1}$, Mohd Basri Mat Nor ${ }^{1}$, Abdul Hadi bin Mohamed ${ }^{1}$

${ }^{1}$ Department of Anaesthesiology and Intensive Care, Kulliyyah of Medicine, International Islamic University Malaysia

Presenter: Iqbalmunauwir bin Ab Rashid

Introduction: Plasma Cystatin $\mathrm{C}$ ( $\mathrm{pCysC}$ ) is one of the functional biomarker for AKI. This study evaluates the utility of $\mathrm{pCysC}$ in diagnosing $\mathrm{AKI}$, predicting death and its correlation with eGFR in septic critically ill patients. Materials and Methods: This is a two centre, prospective observational study of septic critically ill patients. The inclusion criteria were patients older than 18 years old with sepsis, SOFA score of $\geq 2$ and procalcitonin level $\geq 0.5 \mathrm{ng} / \mathrm{ml}$. Serum Creatinine $(\mathrm{sCr})$ and $\mathrm{pCysC}$ were measured at $0,4,24,28,48$, and 52 hours. AKI was defined based on creatinine criteria of the KDIGO guideline. Results: Seventy patients were recruited into this study, of which 32 (45.7\%) had AKI and 15 (21.4\%) died. pCysC diagnosed AKI in all six time intervals with AUC of $0.859,0.858,0.876,0.918,0.887$, and 0.879 for 0 hour, 4, 24, 28, 48, and 52 hours, respectively $(\mathrm{p}<0.0001)$. It did not predict death at any time interval, with an AUC range of 0.053 to 0.608 ( $p>0.1)$. pCysC showed strong negative correlation with all estimates of GFR, with the best profile recorded at 28 hours. Correlation coefficient for eGFR ${ }_{C G}, e G F R_{M D R D}$, eGFR CKD-EPI $_{\text {and }}$ keGFR were $-0.778,-0.763,-0.808$, and -0.781 , respectively $(p<0.0001)$. There is no correlation between cardiac output and $\mathrm{pCysC}$, and eGFR. Correlation coefficient were between -0.208 to 0.267 ( $p>0.1$ ). Conclusion: $p C y s C$ diagnosed AKI in septic critically ill patients and strongly correlated with all estimates of GFR. However, pCysC did not predict death, nor correlate with cardiac output. 